\title{
La gestión de la innovación como la gestión de un ecosistema heterogéneo y estructurado
}

\section{Innovation management as the management of a heterogeneous and structured ecosystem}

\author{
Juan Fernando Arango Londoño ${ }^{1}$ \\ Corona S.A. (Colombia)
}

Recibido el 5 de octubre de 2011, aceptado el 18 de enero de 2012

$\mathrm{N}^{\mathrm{o}}$ de clasificación JEL: O32 y O31

DOI: $10.5295 /$ cdg.110305ja

\section{Resumen:}

Este artículo aborda la innovación como un proceso evolutivo que toma lugar en un ecosistema heterogéneo estructurado. Esto permite entender la gestión de la innovación como la gestión de las interacciones en un ecosistema que se quiere modificar. Inicialmente se presentan las bases del modelo evolutivo y sus mecanismos, y luego se ilustrará la propuesta en un ecosistema que varía en función del aprendizaje, la contrastación del desempeño, y los intereses creencias y valores de los individuos en un marco de acción restringido para el dispositivo edificación sismo-resistente. De forma adicional se identificarán algunas de las interacciones que se deben gestionar para introducir un nuevo dispositivo sismo-resistente en ese ecosistema.

Palabras clave:

Gestión de innovación, evolución, ecosistema, heterogéneo.

\begin{abstract}
:
This paper deals with innovation management as an evolutionary process that take place in a heterogeneous and structured ecosystem. This allows understand Innovation Management as the management of the interactions in a particular ecosystem that is wanted to change. First, theoretical fundamentals for an evolutionary model are presented. Then, an example is given to illustrate the variation of an ecosystem as a function of learning, performance, interests, beliefs and values of the agents. The example also shows agents restrictive action frame when it is wanted to innovate on an "earthquake resistant buildings device" and, also, it identifies the mayor interactions to be managed when a new earthquake resistant device is introduced into that ecosystem.
\end{abstract}

Keywords:

Innovation Management, evolution, ecosystem, heterogeneous.

${ }^{1}$ Corona S.A. (Colombia). Carretera 48 n 72 Sur-01 Sabaneta (Antioquía - Colombia).jfarangol@ corona.com.co; jfarangol@gmail.com.co 


\section{INTRODUCCIÓN}

El acople entre los productos, los procesos, los sistemas y los servicios con la demanda del mercado es uno de los desafíos con mayor exigencia para los gestores de innovación que buscan la competitividad. Para ello, la empresa se apoya en el conocimiento acumulado en productos, procesos, practicas organizacionales y el conocimiento de las necesidades de los usuarios (Fagerberg, 2005, págs. 101-102). Por otra parte se reconoce que las empresas no innovan en aislamiento, sino que lo hacen en interacción extensiva con su ambiente (Fagerberg, 2005, pág. 20). El proceso de innovación involucra por tanto la exploración de oportunidades de productos que respondan a un avance tecnológico, un cambio en la demanda del mercado, o la combinación de éstos. Es por ello que se ha argumentado que la innovación es básicamente un proceso de acople (Kline \& Rosenberg, 1986) (Pavit, 2005, pág. 88). Sin embargo, esto lleva a al problema de qué es primero: las necesidades del mercado, el avance tecnológico, o bien, si lo que ocurre es una especie de sincronía. La respuesta a éste problema parece estar en un marco evolutivo de un ecosistema heterogéneo, en que hay influencias recíprocas en tanto la interacción entre los individuos y los dispositivos transforman el entorno, y el entorno transforma los dispositivos y los individuos.

Por convención algo se considera como una innovación a partir del momento en que se realiza la primera transacción comercial de un nuevo producto, servicio, o la introducción de nuevas fuentes de materias primas, formas organizacionales, o la apertura de nuevos mercados (OECD, 2005) (Freeman \& Soete, 1997). Esto es un asunto de convenciones y contextos que es apropiado para el estudio de los efectos económicos de la innovación, pues da cuenta de los recursos movilizados en el proceso, el nuevo valor agregado, y la dinámica de las empresas. Sin embargo, desde perspectiva de la Gestión de la Innovación, lo que parece más interesante es entender e intervenir la forma en que se introduce en un entorno determinado un nuevo dispositivo, es decir, el proceso por el cual se interviene una red de interacciones determinadas con el fin introducir un cambio ${ }^{1}$.

El proceso de innovación tradicionalmente ha sido presentado a través de teorías y postulados instruccionistas que dictan una serie de pasos o fases de modelos lineales de push, pull o híbridos (Marinova \& Phillmore, 2003). Igualmente, en la forma de postulados en que los esfuerzos de grandes hombres y mujeres que tienen una visión extraordinaria y única del mundo, son quienes logran la innovación. De forma alternativa, se puede entender la innovación como un proceso de adaptación, descrito desde una perspectiva evolutiva de tipo Darwiniano (Nickles, 2003, pág. 57), en que se presentan interacciones reciprocas entre los dispositivos y un entorno, lo que conforma un ecosistema. Por tanto la Gestión de la Innovación podría ocuparse de gestionar las interacciones de los dispositivos existentes, el nuevo dispositivo y su entorno, y tratar de prever los cambios en el entorno.

\footnotetext{
${ }^{1}$ El término dispositivo se refiere a los artefactos, las materias primas, los servicios y las formas organizacionales. En general, los dispositivos se entienden en este contexto como cualquier elemento que ha sido dotado con capacidad de acción o de interacción con otros.
} 


\section{LOS MODELOS EVOLUTIVOS}

El núcleo de la teoría Darwiniana de la selección natural consiste de tres mecanismos básicos: la variación ciega o no dirigida, la selección, y la transmisión o herencia. Estos mecanismos operando en un ambiente razonablemente estable, con presiones de selección, permitiría la evolución de las especies ${ }^{2}$. A pesar de su origen en la biología, la teoría evolutiva de Darwin se ha aplicado a otros campos no necesariamente biológicos, entre otros, el cambio científico, la evolución de la tecnología y la sociedad, los sistemas informáticos, la creatividad y la economía, en los que se resuelven los mecanismos de la teoría de la evolución de diferentes maneras.

En ese sentido, para Toulmin (1972) la adopción de nuevas ideas depende de un proceso de comparación en que se determina si un concepto tiene o no mejor fuerza explicatoria en relación con otros conceptos rivales, dentro de un campo determinado de aplicación. La generación de las ideas se da en los individuos (variación), y en un proceso de discusión y demostración (selección) se demuestra que dicha idea es más provechosa que otras para el propósito buscado. En consecuencia la nueva idea reemplaza a la anterior (o anteriores) y se continúa usando en el tiempo (herencia).

Gabriel Tarde presenta una teoría evolucionista general de los elementos sociales que incluye la innovación. Desde esta perspectiva la invención, es seguida por la oposición y luego por la imitación de aquellos elementos exitosos en competencia con otros ya existentes. Así, los mecanismos de selección operan por competencia entre los nuevos artefactos, pero el proceso se desarrolla con la influencia de factores sociales, por lo cual no se trataría de un proceso puramente evolutivo. Para Tarde, la invención es la fuerza que conduce a la sociedad, pero la sociedad es principalmente imitadora: vestido, moda, simpatía, obediencia y educación. En este caso, la imitación es para la sociedad lo que la herencia es a la biología (Godin, 2008, pág. 25)

Por su parte, en la ingeniería de sistemas los algoritmos evolutivos emulan los procesos de variación ciega y selección. Esto se realiza introduciendo algoritmos genéticos con recombinaciones y mutaciones de carácter aleatorio, en los que se busca la optimización para la solución de un problema mediante la evolución del algoritmo. Otra técnica en los algoritmos genéticos es implementar una búsqueda heurística sistemática, a través de un espacio donde se define un problema que no depende de datos aleatorios, sino de proposiciones if-then en un juego previsible de operaciones y pruebas (Vose, 1999).

En el campo de la psicología, Campbell (1960) propuso que el pensamiento creativo puede ser mejor explicado como un una variación ciega en un proceso de retención selectiva, tomando distancia en que dicho proceso no es una analogía directa con la evolución orgánica $^{3}$. En ese sentido, a diferencia de la evolución biológica la evolución creativa está

\footnotetext{
${ }^{2}$ En otra perspectiva evolucionista, Lamarck (1801), incluyó una ley de desarrollo progresivo para explicar la diferencia entre los seres sencillos y los complejos, así como una ley de generación espontánea para explicar la existencia en el presente de los seres sencillos. La analogía evolutiva para la innovación que exploramos no debería considerar la perspectiva de Lamarck pues supondría aceptar en primera instancia la posibilidad de la generación espontánea de nuevos dispositivos, lo que a su vez supondría aceptar que la innovación se da igualmente de forma espontánea.

${ }^{3}$ Por tanto es un error calificar a Campbell como un estricto Darwinista, por ejemplo (Dasgupta, 2004) (Kronfeldner, In press), entre otros.
} 
afectada entre otros aspectos por la percepción y el aprendizaje. De fondo, tanto en el contexto biológico como en la creatividad, se requiere explicar en primera instancia el origen de las variaciones ciegas y su carácter combinatorio, para luego explicar los procesos de selección y transmisión. De esta forma, tanto la evolución biológica como la creativa dependen de variaciones combinatorias ciegas, pero con significados diferentes en tanto las biológicas son aleatorias y ciegas, mientras que las creativas son únicamente ciegas y no necesariamente aleatorias. Las consecuencias empíricas de este tratamiento de la creatividad es que se conforma un sistema compuesto por el individuo, su dominio y el campo de conocimiento ${ }^{4}$, que permite explicar por ejemplo la productividad, la explosión de información y los descubrimientos múltiples o simultáneos. Así, un campo de conocimiento es una entidad dinámica en tanto que recibe ideas de diferentes individuos, ideas que pueden ser desechadas o aceptadas, pero que en general, la cantidad de ideas aceptadas tiende a hacer que el campo crezca. Sin embargo el sistema opera en un marco estructurado por los procesos creativos de la mente del individuo, las relaciones personales, la relación creatividad-desarrollo de los individuos, y el contexto social ${ }^{5}$. Por otra parte, para Bailey y Cameron (2003), la innovación inicia cuando un individuo presenta una nueva propuesta creativa, que posee novedad y valor en relación con lo existente, lo cual genera tensiones. En este caso, las palabras clave son la estabilidad y el cambio, la novedad y el valor de la novedad. Así, una propuesta que presenta un individuo es evaluada en un entorno o dominio específico, teniendo en cuenta que existen dominios en los cuales las nuevas propuestas son más o menos fácilmente adoptadas.

La economía no ha sido ajena al modelo evolutivo (Hobson, David, entre otros). La idea de una economía evolucionista incorpora las rutinas de las empresas como analogías del comportamiento de los genes en la biología (Nelson \& Winter, 1982). Las rutinas son la base predecible del comportamiento contemporáneo de una empresa, y se heredan en el sentido que se aplican en los nuevos entornos de actuación de la misma, por ejemplo, la investigación y desarrollo de productos, contratación, estrategia, inversiones, portafolio. La búsqueda (mutación) de la modificación de las rutinas, o la incorporación de nuevas rutinas guía a la empresa a un proceso de selección por medio de la evaluación de las utilidades. Finalmente, las nuevas rutinas seleccionadas se incorporan a la empresa (herencia).

\section{LA INNOVACIÓN COMO EVOLUCIÓN}

Así, cualquier sistema viviente o no, en que se puedan combinar los tres mecanismos (variación, selección y transmisión) puede evolucionar ${ }^{6}$, por lo cual estos mecanismos

\footnotetext{
${ }^{4} \mathrm{El}$ aporte del individuo al sistema está compuesto básicamente por las ideas que éste comunica, en forma de productos específicos a un campo de conocimiento como puede ser la química, la física o la biología.

${ }^{5}$ Una presentación de los mecanismos que operan en la creatividad se puede encontrar en (Sternberg, Pretz, \& Kaufman, 2003), y en la habilidad de los individuos para generar o descubrir nuevos problemas en (Root-Bersntein, 2005).

${ }^{6}$ Los mecanismos de variación, selección y transmisión son también tratados como "enactment, selection and retention" o ESR por sus siglas en inglés (Bailey \& Ford, 2003, pág. 251). Enactment en este se refiere a una elección individual que puede ser nueva o rutinaria y que es sensible a los requerimientos del contexto, y que el cambio de un contexto es el resultado de los diferentes enactments que han sido retenidos y seleccionados como una práctica legítima o conmensurable.
} 
no son intrínsecamente procesos de naturaleza puramente biológica (Nickles, 2010). Nickles (2003) ha observado la innovación como un proceso de tipo Darwiniano, en la cual la variaciones y la selección retentiva juegan un papel esencial, sino el único, en la búsqueda de la innovación (Nickles, 2003, pág. 54) ${ }^{7}$. Esto implica que en una primera aproximación, todas las innovaciones, los diseños y el conocimiento, pueden ser construidos como adaptaciones, y en sentido inverso todas las adaptaciones pueden ser construidas como diseño y conocimiento, y cuando son nuevas, como innovaciones (Nickles, 2003, págs. 54-55).

Así, en correspondencia con la teoría de la selección natural, la innovación no fluye directamente de la inferencia lógica, o de la inspiración creativa de un individuo, ni fluye directamente de la nada. Desde esa perspectiva el proceso de innovación es bastante indirecto, pero menos indirecto que la evolución biológica, pues los problemas y metas están bosquejados en sus inicios, y se van modificando durante el proceso de innovación. Por tanto, la innovación como un proceso evolutivo no es totalmente caracterizada por prácticas ciegas de ensayo y error, pero tampoco es un proceso completamente dirigido ${ }^{8}$. En ese mismo sentido, para Achterhuis (1997), la nueva filosofía de la tencología ha encontrado que el desarrollo de la tecnología está acompañado por la transformación de la sociedad, pero por otro lado, que el proceso de desarrollo tecnológico está determinado por factores socio-culturales. Así, en la medida que nuevos artefactos son introducidos en la sociedad, la sociedad es transformada en una forma impredecible e irrevocable, pero igualmente los desarrollos tecnológicos toman forma por determinantes culturales (Achterhuis, 1997, pág. 6).

La denominación usual que se da a los símiles con la evolución biológica es de neo evolutiva, pues la evolución biológica responde a las variaciones genéticas accidentales, al igual que a procesos adaptativos ambientales, lo cual no aplica exactamente para la economía, la creatividad o las ideas. Por ejemplo, el ambiente no hace que el cuello de la jirafa sea largo. En cambio, el cuello de la jirafa es largo por las tendencias de variación genética accidental. Sin embargo en el ambiente se seleccionó ese cuello largo, a manera de adaptación, lo que que incrementó la conservación de esa variación. Esto sería insostenible en la psicología de la creatividad y la innovación, pues la acción del individuo no es solamente accidental sino que está situada en su propio contexto, conocimiento, aprendizaje y representación única del mundo. Esto corresponde a la separación causal de la variación y de la selección que no aplica por ejemplo en la creatividad. En contraste con la analogía biológica, la creatividad de los individuos introduce variaciones que son subsecuentemente aceptadas o rechazadas por las demandas y predilecciones en el contexto donde se realiza la acción ${ }^{9}$, en que el cambio del entorno es el resultado de dichas elecciones.

\footnotetext{
${ }^{7}$ Para Nickles (2003, pág. 55) los procesos evolutivos son los únicos procesos que conocemos que efectivamente llevan a obtener innovaciones.

${ }^{8}$ Esta es una posición no reductivista. Desde una perspectiva puramente Darwinista se requiere algo de ensayo y error, pero no en su totalidad para los procesos de innovación.

${ }_{9}$ Dominio, ambiente y contexto, son empleados de forma intercambiable (Bailey \& Ford, 2003, págs. 248, 251). Utilizaremos el término contexto en correspondencia de nuestra propuesta.
} 


\section{LOS ELEMENTOS DEL ECOSISTEMA}

Tal como se presentó, la analogía evolutiva de Nickles $(2010,2003)$ entrega un buen punto de partida para entender la innovación como un proceso de generación, confrontación y selección retentiva de tecnologías, diseños y conocimiento. Sin embargo, aún es general al no profundizar en cómo operan los mecanismos de generación, selección y retención. Considerar una unidad de análisis donde operan estos mecanismos puede facilitar comprender el proceso de innovación como un proceso neo evolutivo. Proponemos que dicha unidad de análisis es un ecosistema de naturaleza artificial y heterogéneo.

En el sentido más general, un ecosistema natural está integrado por organismos que interactúan en un ambiente. El ambiente en el que vive un organismo contiene los factores externos que lo influencian - tanto los físicos como los químicos-, al igual que contiene otros organismos. El ecosistema se expande a su vez en los individuos, la población (grupos de individuos de la misma especie), y las comunidades (grupo de diferentes poblaciones). (Begon, Townsend, \& Harper, 1986, pág. xi). Por tanto, es posible considerar múltiples ecosistemas, cada uno caracterizado por las interacciones de los diferentes organismos, poblaciones y comunidades, y los factores externos que los afectan.

Así, en una analogía neo evolutiva para la innovación, un ecosistema contiene los individuos humanos, los intereses, creencias y valores de los individuos, el comportamiento de quienes producen los dispositivos, y también los dispositivos no humanos que están cumpliendo ciertas funciones, al igual que las condiciones físicas del entorno. Es posible que existan en ese entorno dispositivos diferentes o similares al nuevo que se pretende introducir. Así, al contener individuos, otros seres vivos y los dispositivos artificiales, el entorno tiene un carácter heterogéneo y artificial en contraste con los ecosistemas naturales (Broncano, 2000). El entorno por su parte está estructurado por las interacciones de los diferentes elementos contenidos en éste que incluyen la orientación de los marcos de actuación para los individuos - en la forma de leyes, normas, elementos éticos y morales y procedimientos aceptados de intervención-, las posibilidades mismas de la naturaleza -la química, la física, la mecánica-, al igual que los dispositivos.

El ecosistema se caracteriza igualmente por ser cambiante. Los intereses, creencias y valores se modifican en el tiempo, al igual que la aplicación de nuevas tecnologías y conocimientos que permiten intervenir el entorno de nuevas formas, con nuevos dispositivos, e incluso intervenir ese entorno de formas totalmente novedosas ${ }^{10}$. A manera de ejemplo, la sostenibilidad ambiental, el interés por los efectos del cambio climático, la interacción en la virtualidad, entre otros, no tenían hace 20 años la prominencia que se observa en nuestros días. Igualmente ha cambiado el interés por la eficiencia y la eficacia energética, industrial, de procesos, o bien la disponibilidad de comunicación permanente de los individuos y la valoración intrínseca del tiempo. Nuevas tecnologías, tales como la nanotecnología, la aplicación de la física cuántica, lo virtual, la multi presencialidad, permiten interactuar en entornos de formas totalmente nuevas. Se puede argumentar que las interacciones características de los diferentes elementos contenidos en ese entorno lo constituyen.

\footnotetext{
${ }^{10}$ Este artículo no pretende sustentar la propuesta por su limitación de extensión. Esto se presenta en la tesis doctoral que desarrolla el autor en la Universidad del País Vasco.
} 
Así, desde la perspectiva de la gestión tecnológica, incorporar un nuevo dispositivo en un ecosistema específico implica acoplarlo en el sistema, es decir, gestionar las interacciones para el nuevo dispositivo. Algunas bondades de este tipo de aproximación en la gestión de la innovación es que permite conciliar el carácter cambiante de los ecosistemas e incluso de la transformación de las innovaciones, pues es un error común tratar la innovación como si fuera algo totalmente definido y homogéneo, como algo que entra en el mercado en un momento preciso (Kline \& Rosenberg, 1986, pág. 283). Permite también entender que un nuevo dispositivo puede acoplarse de forma diferente en diferentes ecosistemas: estos pueden ser micro-entornos tales como la empresa cuando se introducen nuevas materias primas, nuevas formas organizacionales, o bien macro-entornos tales como las regiones, países, o el planeta. De igual forma, explica como diferentes ecosistemas pueden ser más favorables o no para la innovación, en función de marcos de actuación influenciados por políticas regionales, o nacionales. Sin embargo, debe entenderse que es poco probable prever anticipadamente la totalidad de interacciones que un nuevo dispositivo tendrá en un ecosistema, de lo que deriva la complejidad, y que ello siempre implicará incertidumbre y riesgo.

Se pueden plantear así las bases de los mecanismos evolutivos que operan en un ecosistema heterogéneo. Los generadores de una potencial innovación interpretan las interacciones que ocurren en el entorno que pretenden intervenir en función de los intereses creencias y valores de los individuos, los dispositivos existentes y sus propias capacidades tecnológicas y científicas para diseñar nuevos dispositivos (mecanismo de variación). Los nuevos dispositivos se introducen en interacción extensiva con el entorno y entran en competencia con otros dispositivos, siendo evaluados por las personas en función de los intereses, creencias y valores: eficiencia, atributos ecológicos, moda, precio, entre otros. Durante ese proceso de evaluación los nuevos dispositivos pueden ser modificados para ajustar el acople (mecanismo de selección). Así, la innovación no es posible que sea definida de una forma no contextual, es decir, que esto sólo se pude hacer en relación con los otros dispositivos que habitan ese ecosistema, los individuos y sus intereses creencias y valores. Finalmente, los dispositivos que son seleccionados en ese ecosistema son retenidos y se difunden (mecanismo de herencia) y perdurarán en tanto puedan superar exitosamente otros procesos de selección dentro de ese ecosistema. Por tanto, no deben entenderse cada uno de los mecanismos evolutivos como elementos homogéneos y delimitados en el tiempo: En cada uno de los mecanismos, operan internamente para los diferentes componentes del dispositivo sub mecanismos variación, selección y herencia.

\section{UN ECOSISTEMA PARA LAS EDIFICACIONES SISMO-RESISTENTES}

La propuesta de un ecosistema se puede ilustrar en el dispositivo "edificación sismo resistente", el cual tiene entre otras particularidades estar constreñido por un marco de acción, principalmente, en forma de una Ley.

En este caso, el ecosistema está compuesto, de forma no extensiva, por la comunidad de "edificaciones sismo-resistentes" existentes, que coexisten con otros dispositivos similares que no tienen esa capacidad (o no sismo-resistentes), las condiciones físicas de la amenaza sísmica del sitio, los individuos que habitan ese entorno, el conocimiento de los 
mecanismos que generan la amenaza, y el conocimiento de los mecanismos resistentes de las estructuras. A manera de uno de los límites de su ecosistema, debe ser claro que el dispositivo edificación sismo resistente sólo tiene sentido en donde las condiciones físicas del entorno contemplen una fuente cercana de amenaza sísmica. También que en ese entorno habitan individuos y grupos de individuos que razonablemente son conscientes que la amenaza sísmica tiene como consecuencia que su propia vida y su patrimonio están en riesgo, en función de la vulnerabilidad del tipo de dispositivo en que habitan, es decir, si es o no sismo resistente. Gestionar la introducción exitosa de un nuevo dispositivo sismo resistente en ese entorno, es decir, como una configuración nueva de elementos que lleve a una edificación tenga esa característica, requiere no solo la creación del dispositivo, sino que cumpla por ejemplo con los requisitos del marco de acción, uno de los elementos que estructuran y constituyen el ecosistema.

Por ejemplo, la Constitución Nacional de Colombia de 1991 señala que son fines esenciales del Estado garantizar la efectividad de los principios, derechos y deberes consagrados en la Constitución y que las autoridades deben velar por la protección de todas las personas residentes en Colombia en su honra, vida y bienes para asegurar los deberes sociales del Estado y de los particulares. En particular, Colombia está ubicada en una de las regiones más activas sísmicamente en el planeta. Por ello, la protección del Estado en Colombia se extiende más allá de las condiciones de la seguridad y la salud, a la protección ante eventos naturales, entre ellos los sismos, los cuales son previsibles y resistibles. Esto es que la ocurrencia de un sismo, si bien no es susceptible de ser predicha con exactitud en tiempo y lugar, puede ser prevista razonablemente en su magnitud y en la vulnerabilidad de las estructuras, con base en el estudio de los mecanismos generadores de los sismos y los mecanismos resistentes de las estructuras (Asociación Colombiana de Ingeniería Sísmica, 2010).

En este caso, una estructura sismo resistente es un dispositivo tecnológico que está cubierto por una reglamentación específica, en forma de Ley, que “....establece los criterios y requisitos mínimos para el diseño, construcción y supervisión técnica de edificaciones nuevas, así como de aquellas indispensables para la recuperación de la comunidad con posterioridad a la ocurrencia de un sismo, que puedan verse sometidas a fuerzas sísmicas y otras fuerzas de la naturaleza o el uso[...]" (Senado de la República de Colombia, 1997, pág. 1). Esta reglamentación sólo permite construir estructuras con determinados tipos de sistemas sismo resistentes, a saber, muros de carga resistentes a momentos (como es el caso de los muros de hormigón vaciado o los muros en mampostería), pórticos resistentes a momentos (por ejemplo en hormigón o metales), sistema dual (combinación de muros y pórticos resistentes a momentos y cargas laterales), y sistema combinado (pórticos resistentes a cargas verticales combinados con muros o pórticos resistentes a cargas laterales) (Ministerio de Desarrollo Económico, Medio Ambiente y Vivienda, 2010, págs. A52-A58). Otros dispositivos que no son cubiertos por la norma, es decir, novedosos por no ser parte del marco de acción, deben ser acompañados por una autorización de uso que es entregada por la Comisión Asesora de la Norma Sismo Resistente (Senado de la República de Colombia, 1997). Así, el procedimiento para obtener una edificación sismo resistente se convierte en un conjunto de " [...] reglas de procedimiento fundamentadas en el conocimiento científico de las reglas que rigen los materiales y los sistemas físicos concretos con que trabajamos". (Broncano, 2000, pág. 119). Así, las prácticas aceptadas en la ingeniería se re- 
conocen porque tienen la forma de normas o procedimientos estándar, adoptados bien por el Estado, las asociaciones profesionales, u organismos normalizadores, y que se propagan en la formación de los ingenieros y que se constituyen en un marco de acción.

Los mecanismos generadores de los sismos determinan en sentido fuerte la demanda estructural para dicho dispositivo tecnológico, que solo puede ser considerada en forma local en función del área de influencia sísmica para la edificación que es analizada ${ }^{11}$. Es decir, que existen zonas con mayor amenaza sísmica dentro de un país, y aún dentro de éstas zonas, microzonificaciones en donde se atenúan o amplifican los efectos de un sismo.

Inicialmente, se puede observar como el ecosistema va evolucionando, aún sin la introducción de nuevos dispositivos por las interacciones entre los elementos de ese ecosistema. Igualmente cómo el marco de actuación se va modificando. Así, "Dado que la reglamentación sismo resistente corresponde a un documento tecnológico, éste debe actualizarse con alguna periodicidad para plasmar los avances en las técnicas de diseño y las experiencias que se haya tenido con los sismos recientes" (Asociación Colombiana de Ingeniería Sísmica, 2010, pág. i). Así, el énfasis se encuentra en la relación entre la reglamentación, los cambios en la tecnologías de diseño, y en la experiencia en el desempeño de los sistemas permitidos y no permitidos por la misma norma. En ese sentido, el dispositivo "edificación sismo resistente", ubicado en una zonas de amenaza sísmica determinada es evaluada y contrastada en relación con su efectividad de intervención ante episodios sísmicos.

La contrastación de la efectividad del dispositivo tecnológico se evidencia en los registros sísmicos en Colombia que sirven como antecedentes a actualización de la Norma Sismo Resistente de 2010. El sismo de Quindío de magnitud $\mathrm{M}_{\mathrm{L}}=6.2$, ocurrido en enero de 1999, poco más de un año después de la expedición de la NSR-98 de 1998, afectó más de 100000 edificaciones, de las cuales cerca del $80 \%$ eran viviendas y dañó más de de $80 \%$ de las edificaciones educativas, así como la mayoría de instalaciones de salud. Además de los daños materiales, el sismo cobró la vida de 1186 personas, lesionó otras 8500 y 160 000 personas se quedaron sin vivienda. Los daños del evento ascendieron al $1.81 \%$ del PIB de Colombia (Asociación Colombiana de Ingeniería Sísmica, 2010, pág. vii). La severidad de los daños en éste evento sísmico "[...] se explicó en parte, a la superficialidad del evento sísmico y su cercanía a la ciudad, y debido a la amplificación sísmica causada por la presencia de suelos blandos y formas topográficas favorables para el efecto. No obstante, los múltiples daños en edificaciones construidas antes de 1984 ratificaron las enseñanzas de otros sismos en relación con el mal comportamiento de edificaciones frágiles de mampostería no reforzada y de pórticos de concreto reforzado que no cumplían con los requisitos mínimos de sismo resistencia. Se pudo constatar la influencia adversa de elementos no estructurales sobre las estructuras y de las irregularidades de masa y rigidez; aspectos principales por los cuales se había actualizado la reglamentación sismo resistente del país en 1998. En general, muchas edificaciones del centro de la ciudad de Armenia y un número importante de la ciudad de Pereira tuvieron daños estructurales severos debido a la mala calidad de los materiales y debido al mal diseño y a la mala construcción; típica antes de la vigencia de la Reglamentación sismo resistente" (Asociación Colombiana de Ingeniería Sísmica, 2010, pág. vii).

\footnotetext{
${ }^{11}$ La demanda sísmica se refiriere a los movimientos sísmicos de diseño mínimos que se requiere sean resistidos por el dispositivo tecnológico, tales como la aceleración, la velocidad o el desplazamiento del medio transmisor.
} 
En el mismo contexto de aprendizaje y contrastación, el sismo de Pizarro ocurrido en noviembre de 2004, con magnitud $\mathrm{M}_{\mathrm{L}}=6.7$ y epicentro frente a las costas del Océano Pacífico, ocasionó daños sólo en elementos no estructurales y daño mínimo en los elementos estructurales de las edificaciones ubicadas en la ciudad de Cali. Más recientemente, el sismo de Quetame (2008) con magnitud $\mathrm{M}_{\mathrm{L}}=5.7$ provocó el colapso de edificaciones de adobe principalmente en la zona epicentral. Los daños reportados en la ciudad de Bogotá se presentaron en elementos no estructurales y hubo daños mínimos en elementos estructurales (Asociación Colombiana de Ingeniería Sísmica, 2010, págs. viii-ix).

El mecanismo de selección opera aún sin introducir sistemas estructurales novedosos o diferentes a los ya aceptados por la Ley. El desempeño de los dispositivos permitidos y no permitidos es contrastado con su función, en términos de eficiencia y eficacia, pero también en términos de la pérdida de vidas humanas y las pérdidas económicas generadas. En este caso, durante el mecanismo de selección se ratifica que los sistemas estructurales no permitidos por el marco de acción demuestran un mal desempeño, en contraste con aquellos diseñados bajo los parámetros tecnológicos establecidos por el marco de acción. Esto lleva a su vez que se refuerce la no posibilidad de uso de sistemas estructurales que no estén cubiertos por la Ley. Se puede concluir que el dispositivo tecnológico existente en ese ecosistema es contrastado contra el desempeño esperado, al igual que son evaluados los otros dispositivos que no incluyen las características funcionales de los dispositivos sismo resistentes aceptados. Por otra parte, se evidencia como un factor determinante la experiencia en el desempeño de los dispositivos permitidos por la norma en relación con su demanda local de resistencia.

Para este caso, el mecanismo de selección también permite identificar la necesidad de intervenir las prácticas de construcción de los elementos no estructurales a manera de un mecanismo generador de novedades. Es decir, se identifica la necesidad de intervenir el desempeño de aquellos elementos tales como los acabados y los elementos arquitectónicos decorativos, las instalaciones hidráulicas y sanitarias, las instalaciones eléctricas, las instalaciones de gas, los equipos mecánicos (escaleras, ascensores, etc), y las instalaciones especiales que hacen parte de la edificación.

Ahora, si se tratara de gestionar en este ecosistema un nuevo dispositivo no cubierto por la Ley, ¿Qué es lo que se debería gestionar? Los generadores de un nuevo dispositivo para ese ecosistema tienen una idea de una nueva configuración de materiales que permite obtener un dispositivo sismo resistente ${ }^{12}$, en relación directa con las características de ese ecosistema. La nueva idea puede estar mediada por el conocimiento tecnológico y científico de los materiales y la demanda sísmica. Sin embargo, puede ser también bastante indirecta, en la medida que puede ser guiada por otros mecanismos no tecnológicos o empíricos que lleven a la creencia que una nueva configuración dada de materiales permite obtener una resistencia estructural adecuada (mecanismo de variación).

En este caso, la introducción de un nuevo dispositivo sismo resistente comprende la demostración de su buen desempeño, aún antes de ser presentado a los usuarios en razón de

\footnotetext{
${ }^{12}$ En este caso el origen de la idea podría surgir como lo expusimos anteriormente por un mecanismo creativo con mecanismos de retención selectiva, afectada por la percepción y el aprendizaje. La idea para ese ecosistema debe ser novedosa y tener valor en relación con lo existente. Es decir, la idea generalmente incluye una diferenciación positiva en relación con lo que se quiere modificar en ese entorno específico.
} 
los requisitos del marco de acción. Esta demostración se hace con aspectos tecnológicos, tales como el análisis del dispositivo en el laboratorio, su modelación estructural y método de diseño, así como aspectos técnico-legales como es el trámite de un Régimen de Excepción con sus especificaciones técnicas. En el proceso de demostración, el dispositivo puede sufrir transformaciones para ajustarse a las condiciones de desempeño, tales como reforzamientos, cambios en las características de los materiales o conexiones estructurales, o que se acople a los principios del marco de conocimiento común de la ingeniería estructural. Estos cambios operan a manera de nuevos mecanismos de variación, selección y herencia.

El marco de acción establecido en la Ley permite que una vez superados los requerimientos técnicos y legales, el nuevo dispositivo pueda ser implementado, o dicho de otra forma, comercializado (mecanismo de herencia).

Sin embargo, el nuevo dispositivo igualmente será evaluado en relación con otros elementos del ecosistema que no hacen parte del marco de acción restringido, a saber, el precio, las posibilidades arquitectónicas que ofrece, la comodidad, la velocidad constructiva, el número de niveles que puede alcanzar. Podría pensarse que el nivel de gestión de interacción termina allí. Sin embargo, solo basta observar una edificación para entender que el ecosistema para el dispositivo "nuevo tipo de edificación sismo resistente" está compuesto por otras comunidades de dispositivos que también hacen parte de ese ecosistema. Así, se establecen interacciones con otros dispositivos tales como los revestimientos de pisos, paredes y techos, sistemas eléctricos, de calefacción, acueducto, vertimientos de aguas, puertas y ventanas, pinturas y hasta las decoraciones que se adosan a la edificación. A su vez todos estos otros dispositivos han pasado por mecanismos de variación, selección y herencia en relación directa con los otros dispositivos sismos resistentes ya existentes que son diferentes al nuevo dispositivo. La gestión del nuevo dispositivo incorpora la gestión de las interacciones con los demás dispositivos que entrarán en "simbiosis" con éste.

Una vez en uso, el nuevo dispositivo será evaluado y contrastado en relación directa con los daños que puedan presentarse luego de la ocurrencia de otros eventos sísmicos. En ese contexto, si el desempeño del nuevo dispositivo no es satisfactorio, el marco de acción puede restringir totalmente su uso futuro, tal como lo ha hecho con los sistemas estructurales de adobe no reforzado.

\section{CONCLUSIÓN}

Se ha propuesto que la innovación puede ser vista como un proceso evolutivo que opera en un ecosistema heterogéneo y artificial, constituido por las interacciones entre los individuos, los dispositivos y las características del entorno. Así, los cambios en un ecosistema operan bajo mecanismos neo evolutivos darwinianos, mediante mecanismos de variación, selección y retención, donde la extensión del ecosistema puede ser la empresa, una región, un país o global. Esta propuesta permite que la Gestión de la Innovación se ocupe de la gestión de las interacciones en los ecosistemas para introducir cambios, o dicho de otra forma, de acoplar nuevos dispositivos en un sistema que es estructurado. Permite además explicar por qué en algunos ecosistemas en más fácil o no introducir modificaciones, o 
por qué algunos dispositivos son exitosos o no en ciertos ecosistemas, en términos de las interacciones que se establecen.

Así, se plantearon los mecanismos evolutivos que operan en este entorno heterogéneo y artificial. Los generadores de una potencial innovación interpretan las interacciones que ocurren en el entorno que pretenden intervenir en función de los intereses creencias y valores de los individuos, los dispositivos existentes y sus propias capacidades tecnológicas y científicas para diseñar nuevos dispositivos (mecanismo de variación). Los nuevos dispositivos se introducen en interacción extensiva con el entorno y entran en competencia con otros dispositivos, siendo evaluados por las personas en función de los intereses, creencias y valores: eficiencia, atributos ecológicos, moda, precio, entre otros. Durante ese proceso de evaluación los nuevos dispositivos pueden ser modificados para ajustar el acople (mecanismo de selección). Finalmente, los dispositivos que son seleccionados en ese ecosistema son retenidos, se difunden y perdurarán en tanto puedan superar exitosamente otros procesos de selección dentro de ese ecosistema (mecanismo de herencia).

La propuesta se ilustró con el caso del dispositivo "edificación sismo resistente", en que se resaltaron los mecanismos de variación, selección y herencia. Igualmente se planteó que estos mecanismos no son perfectamente homogéneos y definidos, pues en cada uno de ellos operan sub mecanismos que tienen igualmente características evolutivas. Así, en el caso de los dispositivos ya reconocidos como edificaciones sismo resistentes, se mostró como operan mecanismos de variación, selección y herencia, aún sin la introducción de nuevos dispositivos sismo resistentes. Estos mecanismos se presentaron en función de las demandas físicas de un entorno específico en el cual ocurren sismos y en el que este tipo de dispositivos tienen sentido. Igualmente se presentaron los mecanismos evolutivos en función de los intereses y creencias de los individuos, dentro de un marco de acción que en ese caso está fuertemente restringido por una Ley. También se presentó como un nuevo dispositivo no contemplado en ese marco de acción no solo debe ajustarse al marco de acción, sino que requiere interactuar con los otros dispositivos que habitan ese ecosistema. Así, el nuevo dispositivo en ese entorno es generado, seleccionado y heredado, y es objeto de la gestión de la innovación la gestión de las interacciones del nuevo dispositivo en ese ecosistema heterogéneo.

\section{REFERENCIAS BIBLIOGRÁFICAS}

ACHTERHUIS, H. (1997): American Philosophy of Technology: The Empirical Turn, Bloomington (IN), Indiana University Press, pp. 1-10.

ASOCIACIÓN COLOMBIANA DE INGENIERÍA SÍSMICA (2010): "Prefacio", Reglamento colombiano de construccion sismo resistente NSR-10, Bogotá, AIS, pp. i-lxxxi.

BAILEY, J. y FORD, C. M. (2003): "Innovation and Evolution: Managing Tensions Within and Between the Domains of Theory and Practice", The International Handbook on Innovation, Kidlington, Elsevier Ltd., pp. 248-57.

BEGON, M.; TOWNSEND, C. R., y HARPER, J. L. (1986): Ecology: From Individuals to Ecosystems, 4a ed., Oxford, Blackwell Publishing.

BRONCANO, F. (2000): Mundos artificiales-Filosofía del cambio tecnológico, México, Paidós.

CAMPBELL, D. (1960): "Blind Variation and Selective Retention in Creative Thought as in Other Knowledge Processes", Psychological Review, 67, pp. 380-400. 
DASGUPTA, S. (2004): “Is Creativity a Darwinian Process?“, Creativity Research Journal, 16, pp. 403-13.

FAGERBERG, J. (2005): "Innovation: A Guide to the Literature", The Oxford Handbook on Innovation, Oxford, Oxford University Press, pp. 1-27.

FREEMAN, C. y SOETE, L. (1997): The Economics of Industrial Innovation, Cambridge, MIT Press.

GODIN, B. (2008): Innovation: The History of a Category, Project on the Intellectual History of Innovation, UCS, Montreal.

KLINE, S. y ROSENBERG, N. (1986): “An Overview on Innovation”, The Positive Sum Strategy: Harnessing Technology for Economic Growth, Washington, National Academy Press, pp. 275304.

KRONFELDNER, M. (IN PRESS): "Darwinian 'Blind' Hypothesis Formation Revisited”, Synthese.

LAMARCK, J.-B. (1801): Système des animaux sans vertèbres, ou tableau général des classes, des ordres et des genres de ces animaux, Paris, Detreville.

MARINOVA, D. y PHILLMORE, J. (2003): "Models of Innovation”, The International Handbook on Innovation, Kidlington, Elsevier Ltd., pp. 44-53.

MINISTERIO DE DESARROLLO ECONÓMICO, MEDIO AMBIENTE Y VIVIENDA. (2010): "Decreto 926 de 2010, por medio del cual se adopta el Reglamento NSR-10", Reglamento Colombiano de Construcción Sismo Resistente, Bogotá, AIS, pp. H-1.

NELSON, R. y WINTER, S. (1982): An Evolutionary Theory of Economic Change, Cambridge, Harvard College.

NICKLES, T. (2003): "Evolutionary Models of Innovation and the Meno Problem”, The International Handbook on Innovation, Kidlington, Elsevier Ltd., pp. 54-78.

NICKLES, T. (2010): "BVSR as an Abstract Schema for Universal Selection Theory", Physics of Life, pp. 186-187.

OECD. (2005): Guidelines for Collecting and Interpreting Innovation Data -The Oslo Manual-, Paris.

PAVIT, K. (2005): "Innovation Processes", The Oxford Handbook of Innovation, Oxford, pp. 56-114.

ROOT-BERSNTEIN, R. (2005): "Problem Generation and Innovation", The International Handbook on Innovation, Kidlington, Elseviert Ltd., pp. 170-179.

SENADO DE LA REPÚBLICA DE COLOMBIA. (1997): “Ley 400 de 1997”, Normas Colombianas de Diseño y Construcción Sismo Resistente NSR-98, Bogotá, AIS, pp. Ley 400 1-28.

STERNBERG, R.; PRETZ, J. y KAUFMAN, J. (2003): “Types of Innovation”, The International Handbook on Innovation, Kidlington, Elsevier Ltd., pp. 159-169.

TOULMIN, S. (1972): Human Understanding, Oxford, Clarendon Press.

VOSE, M. D. (1999): The Simple Genetic Algorithm, Foundations and Theory, MIT, A Bradford Book. 
